\title{
Chronic Stress Induces Contrasting Patterns of Dendritic Remodeling in Hippocampal and Amygdaloid Neurons
}

\author{
Ajai Vyas,, ${ }^{*}$ Rupshi Mitra, ${ }^{*}$ B. S. Shankaranarayana Rao, and Sumantra Chattarji \\ National Centre for Biological Sciences, Tata Institute of Fundamental Research, Bangalore 560065, India
}

The hippocampus and the amygdala are essential components of the neural circuitry mediating stress responses. The hippocampus, which provides negative feedback regulation of the stress response, is particularly vulnerable to degenerative changes caused by chronic stress. Unlike the hippocampus, relatively little is known about how stress affects the amygdala and the nature of its role in the stress response. Hence, we examined the effects of two different models of chronic stress on hippocampal and amygdaloid neuronal morphology in rats. In agreement with previous reports, chronic immobilization stress (CIS) induced dendritic atrophy and debranching in CA3 pyramidal neurons of the hippocampus. In striking contrast, pyramidal and stellate neurons in the basolateral complex of the amygdala exhibited enhanced dendritic arborization in response to the same CIS. Chronic unpredictable stress (CUS), however, had little effect on CA3 pyramidal neurons and induced atrophy only in BLA bipolar neurons. These results indicate that chronic stress can cause contrasting patterns of dendritic remodeling in neurons of the amygdala and hippocampus. Moreover, CIS, but not CUS, reduced open-arm activity in the elevated plus-maze. These findings raise the possibility that certain forms of chronic stress, by affecting specific neuronal elements in the amygdala, may lead to behavioral manifestations of enhanced emotionality. Thus, stressinduced structural plasticity in amygdala neurons may provide a candidate cellular substrate for affective disorders triggered by chronic stress.

Key words: stress; anxiety; immobilization; rat; hippocampus; CA3; basolateral amygdala; atrophy; hypertrophy; dendritic remodeling
A growing body of evidence has demonstrated that chronic stress can cause hippocampal damage (Uno et al., 1989; McEwen, 1999). Pioneering studies on how stress and stress hormones affect the rat hippocampus revealed that repeated restraint stress produces significant dendritic remodeling in CA3 pyramidal neurons (Watanabe et al., 1992; Magarinos et al., 1996; Sousa et al., 2000). This dendritic remodeling is characterized by a reversible shortening and debranching of apical dendrites (Conrad et al., 1996) and is mediated by mechanisms involving high levels of glucocorticoid secretion and activation of excitatory amino acid release (Magarinos and McEwen, 1995b). These findings have contributed to rodent models of stress-induced neuronal atrophy that may provide one potential explanation for the hippocampal shrinkage associated with post-traumatic stress disorder, recurrent depressive illness, and Cushing's syndrome (Starkman et al., 1992, 1999; Bremner et al., 1995, 1997; Sheline et al., 1996; Lupien et al., 1998).

Over the past decade many studies on stress have focused primarily on the hippocampus, not only because of its susceptibility to stress-related damage but also because of its negative feedback regulation of the stress response via the hypothalamicpituitary-adrenal (HPA) axis (Herman et al., 1989; Jacobson and Sapolsky, 1991; Sapolsky et al., 1991; Herman and Cullinan, 1997). Although the hippocampus is one of the most intensely studied structures in the stress-inhibitory circuit, other limbic

Received Feb. 12, 2002; revised May 2, 2002; accepted May 15, 2002.

This work was supported by research grants from the National Centre for Biological Sciences and Council of Scientific and Industrial Research.

*A.V. and R.M. contributed equally to this work.

Correspondence should be addressed to Dr. Sumantra Chattarji, National Centre for Biological Sciences, UAS-GKVK Campus, Bangalore 560065, India. E-mail: shona@ncbs.res.in.

Copyright (C) 2002 Society for Neuroscience $\quad 0270-6474 / 02 / 226810-\bullet \$ 15.00 / 0$ inputs, which are involved in regulating the HPA axis through excitatory inputs, have received less attention. In particular, there is increasing evidence supporting a critical role for the amygdala in fear, anxiety, and activation of the HPA axis (Allen and Allen, 1974; Davis, 1992; Davis et al., 1994; LeDoux, 1994). Anatomical studies indicate that limbic inputs impinging on the paraventricular nucleus (PVN) of the hypothalamus and hypothalamic GABAergic neurons can be either excitatory from the hippocampus, and thereby enhancing GABAergic tone, or inhibitory from the amygdala, and thereby reducing GABAergic tone (Herman et al., 1989; Jacobson and Sapolsky, 1991; Sapolsky et al., 1991; Pitkanen and Amaral, 1994; Herman and Cullinan, 1997). This in turn implies that although enhanced hippocampal input would suppress the HPA axis, enhanced amygdaloid input could have the opposite effect on HPA activity.

As outlined above, one potential difference between the hippocampus and amygdala with respect to the neural circuitry underlying stress comes from their disparate roles in the regulation of the HPA axis. Evidence for another difference comes from behavioral studies demonstrating how stress affects hippocampalor amygdala-dependent learning and memory. In rodents, stress facilitates aversive learning but impairs spatial learning (Shors et al., 1992; Luine et al., 1994). Although repeated stress that produces dendritic remodeling in the CA3 region impairs hippocampal-dependent learning (Conrad et al., 1996), the basolateral amygdala has been shown to be essential for stress-induced facilitation of aversive learning (Liang et al., 1994; Shors and Mathew, 1998).

In view of the potentially contrasting impact of chronic stress on the hippocampus and amygdala at the behavioral level, and the different roles played by these two structures in the neural circuitry of stress, it is important to examine the effects of chronic 
stress at the level of single neurons. Therefore, we have investigated the effects of two models of chronic stress on the morphology of hippocampal and amygdaloid neurons in rats.

\section{MATERIALS AND METHODS}

Experimental animals. Male Wistar rats were used for chronic unpredictable stress (CUS) and chronic immobilization stress (CIS) protocols. At the beginning of the experiments, CUS animals weighed $200-250 \mathrm{gm}$ (2-2.5 months old) and CIS animals weighed 300-350 gm (3-3.5 months old). All animals (National Centre for Biological Sciences, Bangalore, India) were housed in groups of three with ad libitum access to food and water, unless specified otherwise in stress protocols. Control animals, which were littermates of the stress-treated animals, were housed in separate cages. Animals were maintained in a temperature-controlled room, with a light/dark cycle of $12 \mathrm{hr}$ (lights on at 7:00 A.M.). All procedures related to maintenance and experimentation were in accordance with National Institutes of Health guidelines and approved by the Institutional Animal Ethics Committee.

Experimental treatment groups. Rats, randomly assigned to experimental groups, were subjected to either CIS or CUS for 10 consecutive days. CIS consisted of complete immobilization ( $2 \mathrm{hr} / \mathrm{d}, 10$ A.M.-noon) in rodent immobilization bags without access to either food or water (Nibuya et al., 1999). CUS involved exposing rats to several types of stressors, which varied from day to day, for a period of $10 \mathrm{~d}$ (Ortiz et al., 1996). Thus, for the CUS paradigm, rats were subjected each day to two stressors that were randomly chosen from eight different stressors. The eight stressors were forced swim for 3-4 min, lights on overnight, lights off for $3 \mathrm{hr}$ during the light period of the light/dark cycle, cold stress, social isolation overnight, food and water deprivation overnight, cage movement for $1 \mathrm{hr}$, and immobilization for $1 \mathrm{hr}$. Control animals were not subjected to any type of stress. The following additional parameters were measured to monitor the overall effects of the stress paradigms: percentage gain in body weight (net change in weight after experiment $\times$ 100 /weight at the beginning of experiment), relative adrenal weight (wet weight of adrenal glands in $\mathrm{mg} \times 100$ /body weight in grams), and presence of ulcers on gastric mucosa.

Morphological data analysis. After completion of stress protocols, all groups of rats were killed under deep anesthesia. The brain was removed quickly, and blocks of tissue containing the hippocampus and amygdala were dissected and processed for rapid Golgi staining technique as described earlier (Shankaranarayana Rao et al., 2001). One hemisphere from each brain was used for preparing transverse sections from the dorsal hippocampus, and the other hemisphere was prepared for obtaining coronal sections from the amygdala. For both the hippocampus and amygdala, $120-\mu \mathrm{m}$-thick sections were obtained using a rotary microtome (Jung RM 2055, Leica). Sections were collected serially, dehydrated in absolute alcohol, cleared in xylene, and coverslipped. Slides were coded before quantitative analysis, and the code was broken only after the analysis was completed. To be selected for analysis, Golgi-impregnated neurons had to satisfy the following criteria: (1) presence of untruncated dendrites, (2) consistent and dark impregnation along the entire extent of all of the dendrites, and (3) relative isolation from neighboring impregnated neurons to avoid interfering with analysis.

For morphological quantification of hippocampal neurons, 10 pyramidal neurons (five long-shaft and five short-shaft) from each animal were analyzed from area CA3 of the dorsal hippocampus (Fitch et al., 1989). Three major classes of neurons (pyramidal, stellate, and bipolar/bitufted) from the basolateral complex of the amygdala (BLA) were selected for analysis on the basis of morphological criteria described in the literature (McDonald, 1982; McDonald, 1992). Our analysis of BLA neurons was restricted to those located between bregma $-2.0 \mathrm{~mm}$ and $-3.2 \mathrm{~mm}$, and these were observed to be evenly distributed across the BLA.

Camera lucida tracings $(500 \times)$ were obtained (Leitz Orthoplan) from selected neurons and then scanned (eight-bit grayscale TIFF images with 1200 dpi resolution; HP Scan Jet 6200C) along with a calibrated scale for subsequent computerized image analysis. Custom-designed macros embedded in Object Image software (ftp://simon.bio.uva.nl/pub/, an extended version of NIH Image) were used for morphometric analysis of digitized images. Using the center of the soma as reference point, dendritic length and branch points were measured as a function of radial distance from the soma by adding up all values in each successive concentric segment (Sholl's analysis; segment diameter: $50 \mu \mathrm{m}$ for CA3 pyramidal neurons, $20 \mu \mathrm{m}$ for BLA neurons) (Shankaranarayana Rao et al., 2001).
Elevated plus-maze. The elevated plus-maze, consisting of two opposite open arms $(60 \times 15 \mathrm{~cm}$, surrounded by 1 -cm-high transparent wall $)$ and two enclosed arms $(60 \times 15 \mathrm{~cm}$, surrounded by a $15-\mathrm{cm}$-high opaque wall), was elevated $75 \mathrm{~cm}$ from ground. Individual trials lasted for $5 \mathrm{~min}$ each and were recorded with a video camera for off-line analysis. At the beginning of each trial, animals were placed at the center of the maze, facing an enclosed arm. All trials were conducted between 10 A.M. and 2 P.M., and the maze was cleaned with $5 \%$ ethanol solution (v/v) after each trial.

Statistical analysis. Statistical significance for the effects of CUS and CIS on dendritic branch points and dendritic length of CA3 pyramidal neurons were analyzed by one-way ANOVA. Because values for dendritic branch points and dendritic length of BLA neurons did not conform to a normal distribution, a more rigorous and stringent nonparametric statistical analysis involving a distribution-free randomized Mann-Whitney test (Potvin and Roff, 1993) was applied to evaluate levels of significance of morphological changes in the amygdala.

\section{RESULTS}

\section{Effects of chronic immobilization stress on dendritic morphology of hippocampal CA3 pyramidal neurons}

CIS caused a significant decrease in the dendritic length $(p<$ $0.01)$ and the number of branch points $(p<0.01)$ in hippocampal CA3 pyramidal neurons as compared with neurons in control animals (Table 1). Both long-shaft and short-shaft CA3 pyramidal cells showed significant dendritic atrophy after CIS. Moreover, the decrease in total dendritic length and number of branch points was evident in both apical as well as basal dendrites of CA3 pyramidal cells. The atrophy of basal dendrites, however, was not as pronounced as that observed in apical dendrites (Table 1). Representative camera lucida drawings of control and CIS longshaft CA3 neurons are depicted in Figure $1 B$.

To investigate the effects of CIS in greater detail, a segmental analysis was performed to track the changes in dendritic length and branch points as a function of radial distance from the cell soma (Fig. 1A). This analysis demonstrates that CIS induced the most pronounced reduction in both apical dendritic length and number of apical branch points of long-shaft CA3 neurons within a distance of 150-250 $\mu \mathrm{m}$ from the soma. Basal dendritic length was reduced most significantly within the first $150 \mu \mathrm{m}$ from the soma, whereas the most significant decrease in number of basal branch points (Fig. $1 \mathrm{~A}$, bottom right panel) occurred within $50 \mu \mathrm{m}$ of the soma. Short-shaft CA3 neurons exhibited a similar pattern of atrophy after CIS (data not shown).

\section{Effects of chronic unpredictable stress on dendritic morphology of CA3 pyramidal neurons}

In contrast to CIS, CUS was not as effective in causing dendritic atrophy in CA3 pyramidal neurons (Table 1). We observed several points of difference between the effects induced by CIS and CUS. First, the magnitudes of the reduction in total dendritic length and the number of branch points in CUStreated long-shaft CA3 neurons were considerably smaller compared with those elicited by the CIS paradigm. For example, the average percentage change in total apical dendritic length ( $-13 \%$ from control) (Table 1) and number of apical branch points ( $-13 \%$ from control) (Table 1$)$ induced by CUS was less than half of their corresponding values after CIS (total apical dendritic length: $-29 \%$ from control; number of apical branch points: $-31 \%$ from control) (Table 1). Second, these changes did not have the same degree of statistical significance compared with CIS-induced changes in the same variables. Third, only apical, but not basal, dendrites of long-shaft CA3 cells showed a significant $(p<0.05)$ reduction in both total dendritic length and number of branch points. Finally, al- 


\begin{tabular}{|c|c|c|c|c|c|c|}
\hline & \multicolumn{3}{|c|}{ Chronic immobilization stress (CIS) } & \multicolumn{3}{|c|}{ Chronic unpredictable stress (CUS) } \\
\hline & Control & CIS & $\%$ Change & Control & CUS & $\%$ Change \\
\hline Long shaft CA3 neurons & $(n=50)$ & $(n=45)$ & & $(n=45)$ & $(n=50)$ & \\
\hline \multicolumn{7}{|l|}{ Dendritic length } \\
\hline Apical & $2113 \pm 65$ & $1498 \pm 59^{* *}$ & -29 & $2014 \pm 102$ & $1749 \pm 63^{*}$ & -13 \\
\hline Basal & $1827 \pm 49$ & $1527 \pm 64^{* *}$ & -16 & $1808 \pm 102$ & $1740 \pm 60$ & NS \\
\hline \multicolumn{7}{|l|}{ Branch points } \\
\hline Apical & $14.7 \pm 0.5$ & $10.2 \pm 0.3^{* *}$ & -31 & $14.0 \pm 0.6$ & $12.2 \pm 0.5^{*}$ & -13 \\
\hline Basal & $16.2 \pm 0.5$ & $13.6 \pm 0.4^{* *}$ & -16 & $16.2 \pm 0.8$ & $15.2 \pm 0.5$ & NS \\
\hline Short shaft CA3 neurons & $(n=55)$ & $(n=45)$ & & $(n=45)$ & $(n=55)$ & \\
\hline \multicolumn{7}{|l|}{ Dendritic length } \\
\hline Apical & $2249 \pm 76$ & $1746 \pm 66^{* *}$ & -22 & $2236 \pm 124$ & $1916 \pm 68^{*}$ & -14 \\
\hline Basal & $1850 \pm 56$ & $1572 \pm 60^{* *}$ & -15 & $1832 \pm 82$ & $1585 \pm 42^{* *}$ & -13 \\
\hline \multicolumn{7}{|l|}{ Branch points } \\
\hline Apical & $15.7 \pm 0.6$ & $12.4 \pm 0.4^{* *}$ & -21 & $16.9 \pm 0.7$ & $14.4 \pm 0.5^{* *}$ & -15 \\
\hline Basal & $15.5 \pm 0.4$ & $13.5 \pm 0.6^{* *}$ & -13 & $15.7 \pm 0.8$ & $13.3 \pm 0.4^{* *}$ & -15 \\
\hline
\end{tabular}

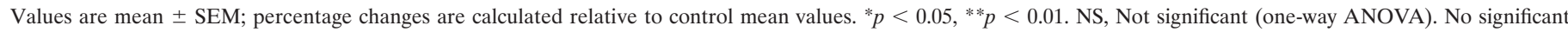
$(p>0.1)$ difference between mean values for CIS-control and CUS-control neurons.

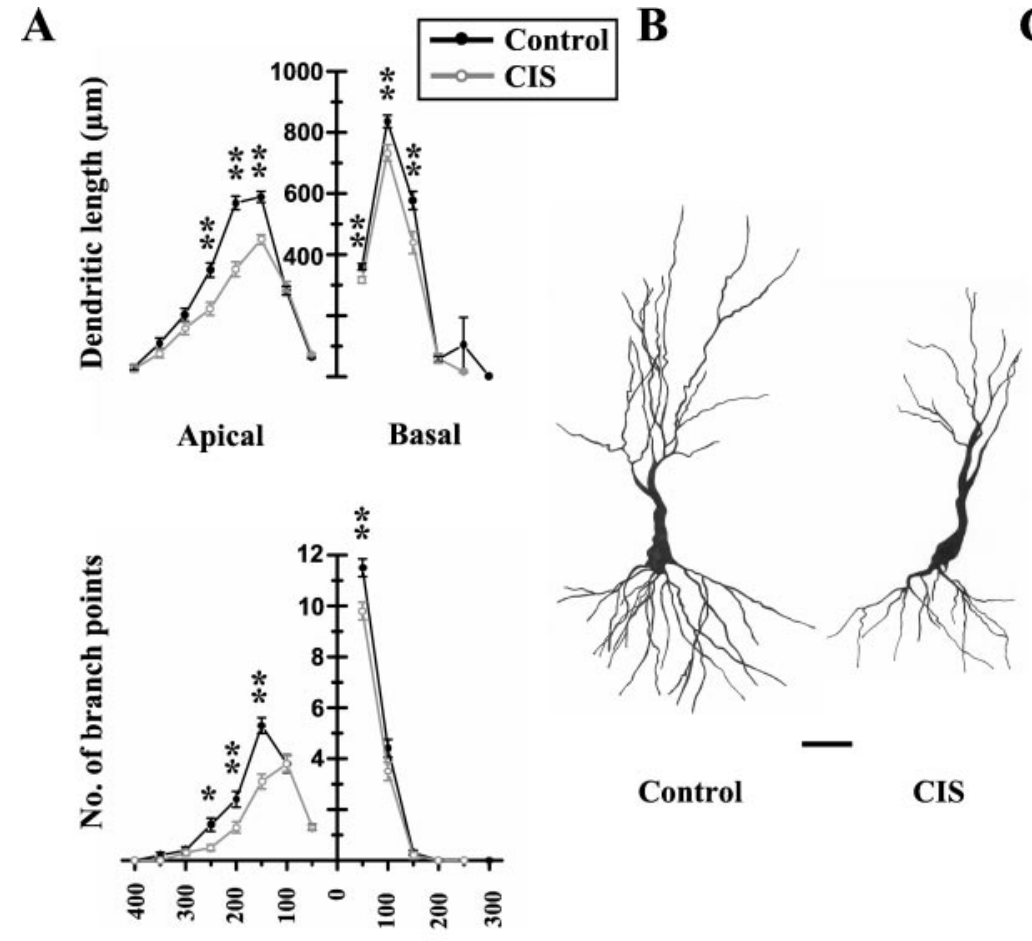

Radial distance from soma $(\mu \mathrm{m})$
$\mathrm{C}$

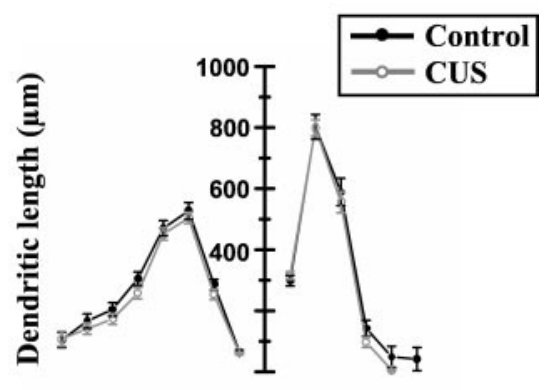

Apical

Basal

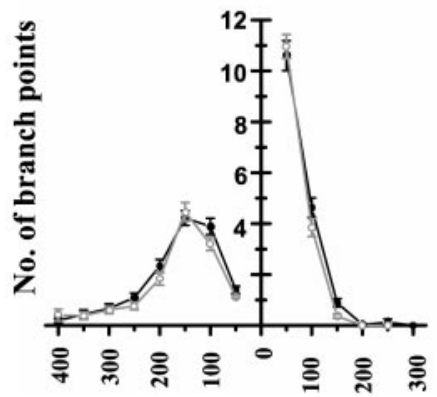

Radial distance from soma $(\mu \mathrm{m})$

Figure 1. CIS is more effective compared with CUS in causing dendritic atrophy in hippocampal long-shaft CA3 pyramidal neurons. $A$, Effects of CIS on mean dendritic length (top) and number of branch points (bottom) for each successive $50 \mu \mathrm{m}$ segment as a function of the radial distance of the corresponding segment from the soma (control cells, $n=50$; CIS cells, $n=45$ ). Changes in apical (left) and basal (right) dendrites are shown separately. $B$, Camera lucida drawings of representative Golgi-impregnated hippocampal CA3 pyramidal neurons from control and CIS-treated animals. Scale bar, $50 \mu \mathrm{m}$. $C$, Effects of CUS on mean dendritic length (top) and number of branch points (bottom) for each successive $50 \mu \mathrm{m}$ segment as a function of the radial distance of the corresponding segment from the soma (control cells, $n=45$; CUS cells, $n=50)$. Changes in apical (left) and basal (right) dendrites are shown separately. ${ }^{*} p<0.05,{ }^{* *} p<0.01$; one-way ANOVA. Filled circle, black line: Control; open circle, gray line: CIS or CUS.

though short-shaft CA3 neurons exhibited atrophy in both apical and basal dendrites, the magnitudes of the reduction in total dendritic length and number of branch points were almost identical (Table 1). In contrast, CIS-induced atrophy in apical dendrites was relatively greater compared with basal dendrites (Table 1).
The relatively smaller effects induced by CUS compared with CIS become clearly evident in the more detailed segmental analysis presented in Figure $1 C$. Unlike the significant CIS-induced atrophy observed at radial distances of 150-250 $\mu \mathrm{m}$, CUS failed to elicit a comparable effect at any distance from the soma. 
Table 2. Effects of CIS and CUS on total dendritic length $(\mu \mathrm{m})$ of BLA neurons

\begin{tabular}{llllll} 
& \multicolumn{2}{l}{$\begin{array}{l}\text { Chronic immobilization } \\
\text { stress (CIS) }\end{array}$} & & \multicolumn{2}{l}{$\begin{array}{l}\text { Chronic unpredictable } \\
\text { stress (CUS) }\end{array}$} \\
\cline { 2 - 3 } \cline { 5 - 6 } & Control & CIS & & Control & CUS \\
\hline Pyramidal & $1330(699)$ & $1666(761)^{*}$ & & $1822(908)^{\dagger}$ & $1712(658)$ \\
Stellate & $1515(501)$ & $1715(779)^{*}$ & & $1771(480)^{\dagger}$ & $1626(677)$ \\
Bipolar & $1121(524)$ & $1149(509)$ & & $1351(833)^{\dagger}$ & $1174(484)^{*}$
\end{tabular}

$\overline{\text { Values are median (inter-quartile range); }{ }^{*} p<0.05 \text {, CIS or CUS versus respective }}$ controls (randomized Mann-Whitney test); ${ }^{\dagger} p<0.05$, CIS-control versus CUScontrol neurons (randomized Mann-Whitney test).

\section{Effects of chronic immobilization stress on dendritic morphology of amygdala neurons}

Having established the overall efficacy of our chronic stress protocols in eliciting patterns of dendritic atrophy in hippocampal CA3 pyramidal neurons that are qualitatively similar to those reported previously, we next analyzed morphological effects of CIS on Golgi-impregnated amygdala neurons in the same animals. Changes in dendritic length and number of branch points in control and CIS-treated neurons in the BLA were analyzed using the same methods applied to the hippocampal neurons.

Previous morphological studies have revealed that the cortexlike BLA contains two main cell-types: spiny pyramidal (or modified pyramidal) neurons and spine-sparse nonpyramidal neurons (McDonald, 1982, 1992). Amygdaloid "pyramidal neurons" constitute a broad, continuous morphological spectrum, from neurons that are virtually identical to cortical pyramidal neurons at one end to neurons that more closely resemble cortical spiny stellate cells at the other end of the spectrum. These studies also suggest that as in the cerebral cortex, it is possible to recognize bitufted/bipolar varieties of nonpyramidal cells on the basis of dendritic arborization patterns. Our analysis, therefore, used this framework (McDonald, 1982, 1992) to analyze morphological effects of the CIS paradigm on three classes of BLA neurons: pyramidal, stellate, and bitufted/bipolar.

The same CIS paradigm that caused dendritic atrophy in CA3 pyramidal neurons in the hippocampus induced the opposite effect in BLA pyramidal neurons (Table 2). We observed a significant $(p<0.05)$ increase in dendritic length of CIS-treated pyramidal neurons [median(inter-quartile range) $=1666(761)$ $\mu \mathrm{m}$ ] compared with control pyramidal neurons [median(interquartile range $)=1330(699) \mu \mathrm{m}]$ (Fig. $2 C)$. This increase $(25 \%$ compared with control) in median dendritic length was investigated further using segmental analysis, and the results are presented in Figure $2 A$. Segmental analysis in incremental steps of $20 \mu \mathrm{m}$ from the soma clearly shows that dendritic length of CIS-treated BLA neurons underwent the most pronounced increase within a distance of 60-160 $\mu \mathrm{m}$ from the soma (Fig. 2A). In this particular range of radial distance from the pyramidal cell soma, all dendritic length median values for the CIS-treated neurons were above the 75 th percentile value for control neurons. The total number of branch points was also greater in CIS-treated pyramidal neurons [median(inter-quartile range) $=15.0(3.2)$ ] compared with control neurons [median(inter-quartile range) = 13.5(4.2)] (Fig. 2C). Representative camera lucida drawings of BLA pyramidal neurons for control and CIS animals are depicted in Figure $2 B$.

Similar to the morphological changes exhibited by pyramidal neurons after CIS, BLA stellate neurons (Fig. 3B) also showed an
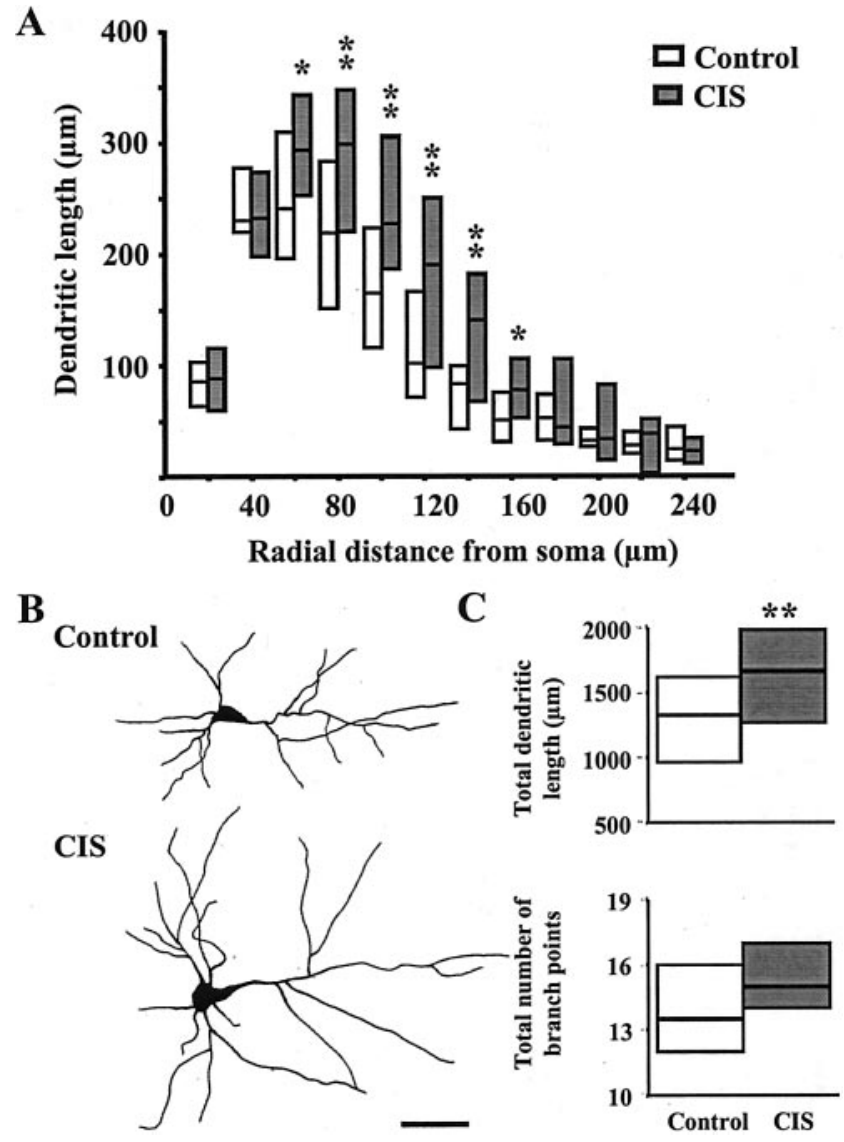

Figure 2. CIS increases dendritic arborization in BLA pyramidal neurons. $A$, Median values (horizontal line within each vertical bar) of total dendritic length for each successive $20 \mu \mathrm{m}$ segment as a function of the radial distance of the corresponding segment from the soma (control cells, $n=18$; CIS cells, $n=22$ ). Inter-quartile ranges are represented by the lower (25th percentile) and upper ( 75 th percentile) bounds of each vertical bar. B, Camera lucida drawings of representative Golgi-impregnated BLA pyramidal neurons from control and CIS-treated animals. Scale bar, $50 \mu \mathrm{m}$. $C$, Plots of median values and inter-quartile ranges for total dendritic length (top) and total number of branch points (bottom) for control $(n=18)$ and CIS $(n=22)$ neurons. ${ }^{*} p<0.05, * * p<0.01$; randomized Mann-Whitney test.

increase in both total dendritic length and total number of branch points (Table 2). Segmental analysis (Fig. $3 A$ ) reveals that the most significant and pronounced increase in dendritic length occurred within a distance of $60 \mu \mathrm{m}$ from the soma. Furthermore, even in segments that did not exhibit a statistically significant difference, the CIS-treated stellate neurons tended to have higher median values relative to control neurons. In contrast to pyramidal and stellate neurons, bipolar/bitufted neurons were not affected by CIS (Table 2). The overall efficacy of the CIS protocol in inducing dendritic remodeling was also analyzed by assessing its impact on the entire population for each of the three classes of BLA neurons. Cumulative frequency plots (see Fig. $5 A$ ) of the entire database obtained from all three classes of BLA neurons clearly demonstrate that neurons with a wide range of dendritic lengths showed the same trends as their respective median values. This is particularly evident in BLA pyramidal neurons, where the increase in total dendritic length was evenly distributed across neurons with a wide range of dendritic lengths and was comparable to the increase shown by the median value for the distribution. Thus, in contrast to the lack of effect in the bipolar/bitufted 


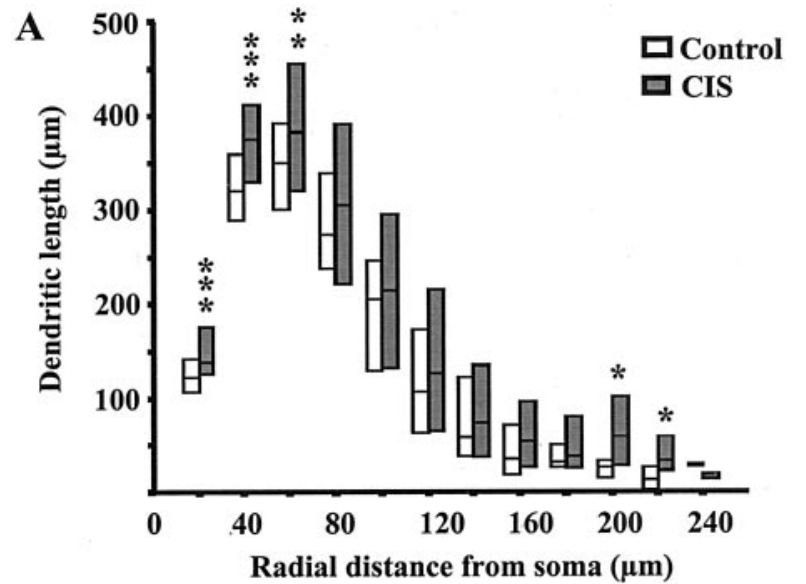

B

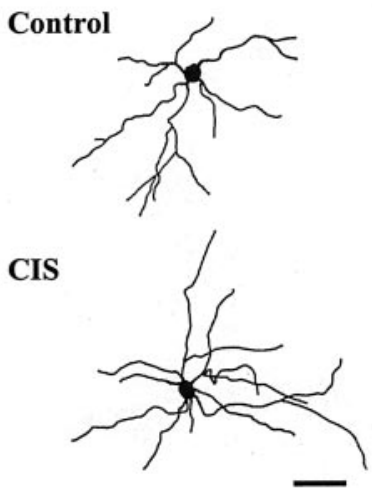

C
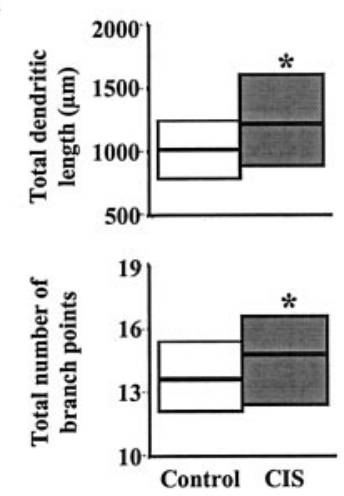

Figure 3. CIS increases dendritic arborization in BLA stellate neurons. $A$, Median values (horizontal line within each vertical bar) of total dendritic length for each successive $20 \mu \mathrm{m}$ segment as a function of the radial distance of the corresponding segment from the soma (control cells, $n=$ 43; CIS cells, $n=39$ ). Inter-quartile ranges are represented by the lower (25th percentile) and upper (75th percentile) bounds of each vertical bar. $B$, Camera lucida drawings of representative Golgi-impregnated BLA stellate neurons from control and CIS-treated animals. Scale bar, $50 \mu \mathrm{m}$. $C$, Plots of median values and inter-quartile ranges for total dendritic length (top) and total number of branch points (bottom) for control $(n=$ $43)$ and CIS $(n=39)$ neurons. ${ }^{*} p<0.05, * * p<0.01,{ }^{* * *} p<0.001$; randomized Mann-Whitney test.

neurons, CIS-induced increase in dendritic length appears to be occurring across the entire population of pyramidal neurons analyzed for the present study.

\section{Effects of chronic unpredictable stress on dendritic morphology of amygdala neurons}

The same three classes of BLA neurons that were studied in the CIS experiments were also analyzed after CUS. In contrast to the CIS-induced effects, the morphological changes exhibited by BLA neurons following the CUS paradigm were quite different. First, the CUS paradigm only affected bipolar/ bitufted neurons in the BLA (Table 2). Second, unlike the increase observed in dendritic parameters of pyramidal and stellate neurons after CIS, CUS caused a significant decrease $(p<0.05)$ in total dendritic length (Table 2). Table 2 also shows a significant difference in median dendritic length between control neurons of the CIS and CUS groups. This may be attributed to the age difference between animals used in the two stress protocols.

Segmental analysis of CUS-induced dendritic atrophy of bipolar/bitufted neurons (Fig. $4 A$ ) reveals that throughout the entire
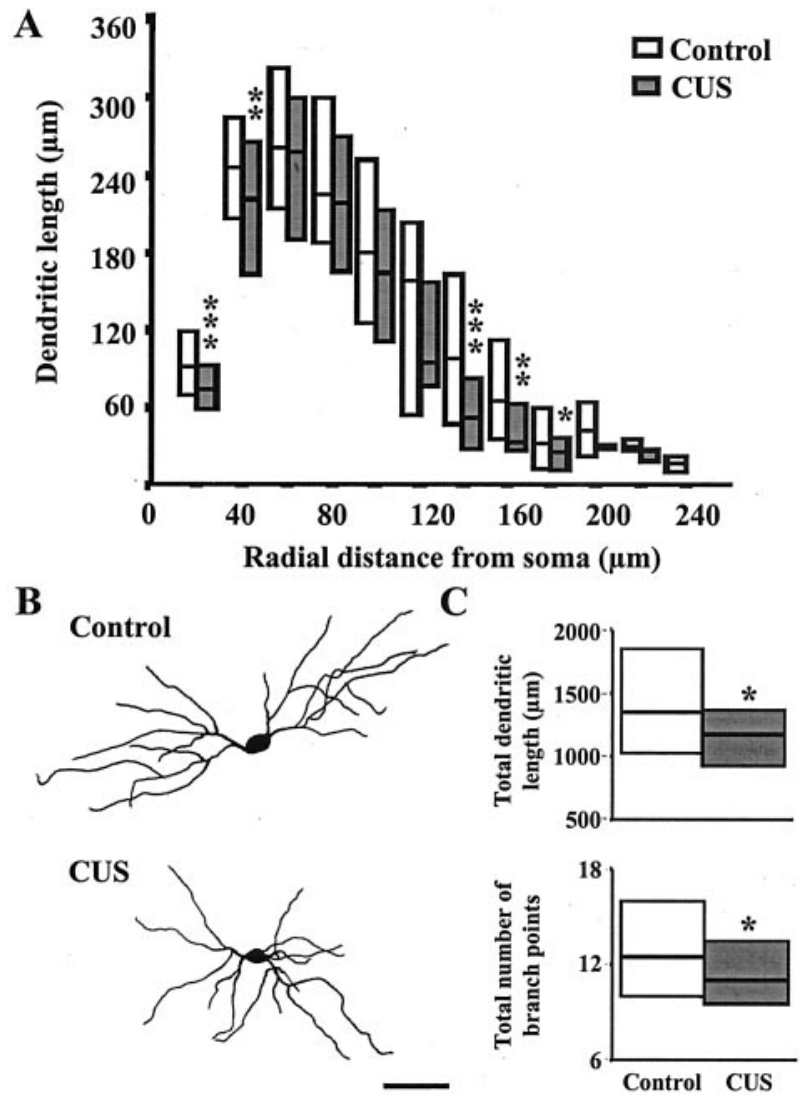

Figure 4. CUS causes dendritic atrophy in BLA bipolar/bitufted neurons. $A$, Median values (horizontal line within each vertical bar) of total dendritic length for each successive $20 \mu \mathrm{m}$ segment as a function of the radial distance of the corresponding segment from the soma (control cells, $n=34$; CUS cells, $n=39$ ). Inter-quartile ranges are represented by the lower (25th percentile) and upper ( 75 th percentile) bounds of each vertical bar. B, Camera lucida drawings of representative Golgi-impregnated BLA bipolar/bitufted neurons from control and CUS-treated animals. Scale bar, $50 \mu \mathrm{m}$. $C$, Plots of median values and inter-quartile ranges for total dendritic length (top) and total number of branch points (bottom) for control $(n=34)$ and CUS $(n=34)$ neurons. ${ }^{*} p<0.05$, ${ }^{*} * p<0.01$, $* * * p<0.001$; randomized Mann-Whitney test.

extent of the dendritic tree, the median values for the dendritic length for any particular segment were always smaller than the corresponding control values. The most significant reduction in dendritic length was evident within a distance of $40 \mu \mathrm{m}$ from the soma, as well as at a distance of $140-180 \mu \mathrm{m}$ from the soma (Fig. $4 A$ ). The lack of any CUS-induced effects on BLA pyramidal and stellate neurons was borne out across the entire range of values for total dendritic length (Fig. 5B).

\section{Effects on body and adrenal weights}

To compare the indices of dendritic remodeling with other measures of the effects of chronic stress, we monitored relative gain in body weight and relative adrenal weight. Percentage body weight gain was significantly ( $p<0.001$; Student's $t$ test) reduced after completion of the $10 \mathrm{~d}$ stress protocol for both CIS (CIS: $-0.2 \pm$ $1.2 \%, n=34$; control: $6.4 \pm 1.2 \%, n=36$ ) and CUS (CUS: $2.7 \pm$ $2.0 \%, n=32$; control: $15.1 \pm 1.3 \%, n=25$ ) animals. Interestingly, only CIS caused significant adrenal hypertrophy (relative adrenal weight, CIS: $15.9 \pm 0.8, n=34$; control: $13.6 \pm 0.7, n=$ 34; $p<0.05$; Student's $t$ test). 


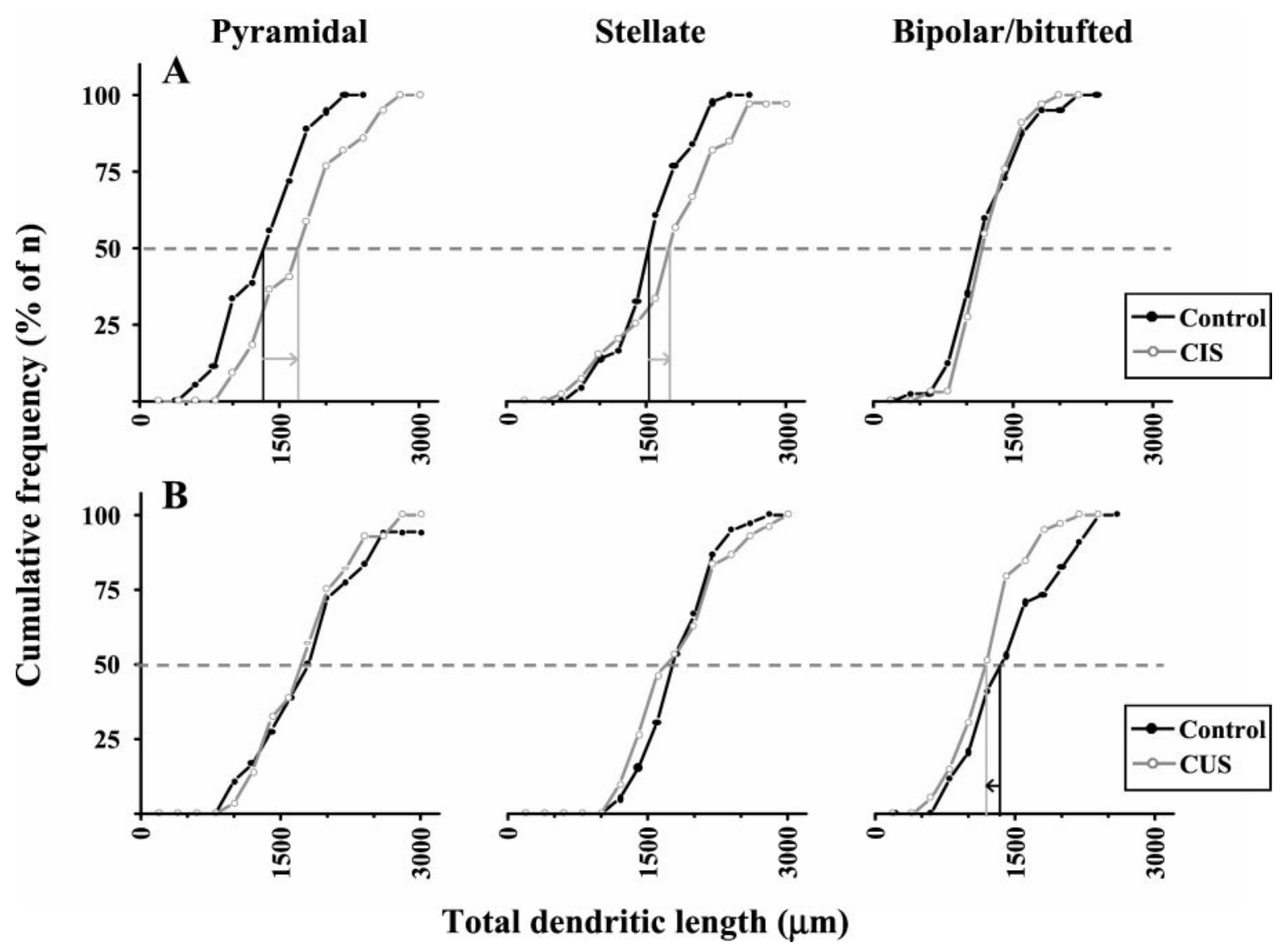

Figure 5. Cumulative frequency plots summarizing the data for total dendritic length illustrating the contrasting effects of CIS $(A)$ and CUS ( $B)$ on all BLA neurons. Pyramidal, left column; stellate, middle column; bipolar/bitufted, right column. The $50 \%$ mark (dashed line) for the total $n$ in each plot represents the median value for the total dendritic length for the corresponding neuronal class. For clarity, the median values are marked with a solid vertical line (control: black; CIS or CUS: gray) in only those cases where there is a statistically significant difference relative to control. CIS induces a significant increase (right arrow) in total dendritic lengths of pyramidal and stellate neurons, without significant effect on bipolar/bitufted neurons. CUS causes a significant decrease (left arrow) in total dendritic length of bipolar/bitufted neurons, without affecting pyramidal and stellate neurons. Filled circle and black line: Control; open circle and gray line: CIS or CUS.

\section{Anxiety response after chronic immobilization and unpredictable stress}

Previous studies suggest that repeated restraint stress can have powerful enhancing effects on emotionality (Conrad et al., 1999). Animals display distinct behavioral changes suggestive of an anxiety response after exposure to stress. Therefore, the behavioral response of CIS and CUS animals in an anxiogenic environment was investigated using the elevated plus-maze and compared with control animals. CIS animals exhibited a significant ( $p<0.05$; Student's $t$ test) reduction in both percentage open-arm entries (CIS: $24.9 \pm 5.9 \%, n=10$; control: $40.9 \pm 3.6 \%, n=10)$ and percentage time in open arms (CIS: $12.2 \pm 4.6 \%, n=10$; control: $21.6 \pm 3.3 \%, n=10)$. Thus, the CIS animals made fewer entries and spent less time in the open arms of the maze than control animals, indicative of an enhanced anxiety response. In contrast, no significant effects were observed in CUS animals for percentage open-arm entries $(39.2 \pm 6.4 \% ; n=10)$ and percentage time in the open arms $(25.5 \pm 5.5 \% ; n=10)$. We conclude that CIS induced significantly greater anxiety compared with CUS and control animals (Vyas et al., 2001).

\section{DISCUSSION}

This study explored two aspects of how chronic stress affects the hippocampus and amygdala. First, our goal in these experiments was to see whether chronic stress induces morphological changes in the amygdala and how they compare with those observed in the hippocampus (Watanabe et al., 1992). Second, we tested whether these stress-induced morphological changes follow the same general pattern across two very different regimens of chronic stress, CIS and CUS (Ortiz et al., 1996; Nibuya et al., 1999). Our findings demonstrated that chronic stress induces contrasting patterns of dendritic remodeling in hippocampal and amygdaloid neurons. CIS elicited significant dendritic atrophy in hippocampal CA3 pyramidal neurons, but caused dendritic hypertrophy in BLA neurons. This CIS-induced enhancement in dendritic arborization was restricted only to BLA pyramidal and stellate neurons, which are presumably excitatory projection neurons (McDonald, 1982, 1992). Moreover, these patterns varied, depending on the type of chronic stress used. CUS, which only caused atrophy, was relatively less effective in remodeling CA3 pyramidal neurons and specifically affected BLA bipolar/bitufted neurons. Finally, CIS and CUS also differed in terms of their anxiogenic properties because CIS, but not CUS, reduced open-arm activity in the elevated plus-maze.

\section{CIS is more effective than CUS in inducing dendritic atrophy in CA3 pyramidal neurons}

Previous studies of the hippocampus as a target of stress and stress hormones have characterized essential features of struc- 
tural remodeling in CA3 pyramidal neurons. The earliest reports established a rat model that demonstrated that $21 \mathrm{~d}$ of chronic restraint stress $(6 \mathrm{hr} / \mathrm{d})$ or $21 \mathrm{~d}$ of corticosterone treatment caused apical dendrites of CA3 pyramidal neurons to decrease in length and branching (Watanabe et al., 1992; Magarinos and McEwen, 1995b). This pattern of apical dendritic atrophy was also observed in the tree shrew after chronic psychosocial stress (Magarinos et al., 1996). The present study demonstrates that CIS for $10 \mathrm{~d}$ (2 $\mathrm{hr} / \mathrm{d}$ ) is capable of inducing a pattern of shortening and debranching of dendrites in hippocampal CA3 pyramidal neurons that is consistent with previous findings. Interestingly, although earlier studies have reported atrophy only in apical dendrites, our results indicate that CIS caused significant atrophy in basal dendrites as well. Complete immobilization, considered to be more severe compared with restraint stress, may be more effective in eliciting structural changes in both apical and basal dendrites. However, the degree of shortening and debranching induced by CIS was generally more pronounced in apical dendrites relative to basal dendrites (Table 1).

Our findings also suggest that CUS was less effective compared with CIS in its ability to elicit dendritic atrophy (Table 1). Although repeated restraint stress has commonly been used in past studies, there is a growing appreciation of the fact that repeated application of the same stressor can lead to habituation in the stress response (Melia et al., 1994). Therefore, stress protocols, comprising a combination of different stressors, have been used to reduce such adaptive effects (Ortiz et al., 1996). Studies using repeated unpredictable stress have been shown to elicit behavioral and biochemical changes that have not been observed with repeated predictable stress (Sapolsky et al., 1984), but there is also evidence (Magarinos and McEwen, 1995a) indicating that repeated restraint stress and a chronic multiple stress paradigm produced the same degree of apical dendritic atrophy in CA3 pyramidal neurons despite differences in terms of the degree and time course of non-neural measures, e.g., habituation of the corticosterone response to acute restraint stress, body weight gain, thymus atrophy, and adrenal gland hypertrophy. This suggests that mediators in addition to adrenal steroids can influence the time course of an array of stress indices in a complex manner. It may well be that not all of the individual stressors used in our CUS paradigm work in concert to trigger similar temporal variations in stress indices that can lead to a robust morphological phenotype. In the present study, although both CIS and CUS were comparable in terms of reduced body weight gain, the CIS paradigm produced greater adrenal hypertrophy. This, in turn, is consistent with the greater magnitude of dendritic atrophy induced by CIS.

\section{CIS enhances dendritic arborization in BLA pyramidal and stellate neurons}

Having established the efficacy of our CIS regimen in producing morphological effects that match previous reports on the hippocampus, the novel and interesting observations of the present study came from our analyses of stress-induced morphological changes in the amygdala. The observed dendritic growth in the BLA is particularly interesting for several reasons. First, although pyramidal neurons appear to be one of the prime targets of the CIS-induced morphological changes in both hippocampus and amygdala, the changes were in opposite directions (atrophy vs hypertrophy). Second, the enhanced dendritic arborization in the BLA was not manifested uniformly across all neuronal classes (Fig. 5) because bipolar/bitufted neurons remained unaffected by
CIS. Third, only the bipolar/bitufted BLA neurons were affected by CUS, and that too in the opposite direction compared with the CIS-induced hypertrophy. In other words, there are multiple levels of dissociation in our observations leading us to conclude that the two regimens of chronic stress have rather specific and contrasting effects within the hippocampal and amygdaloid circuitry.

On the basis of morphological studies, the BLA, which is described as a cortex-like nucleus of the amygdala (Swanson and Petrovich, 1998), has been shown to contain a large number of spiny excitatory projection neurons, which are "pyramidal-like" (McDonald, 1982, 1992). According to recent studies characterizing the electrophysiological properties of morphologically identified amygdala neurons, pyramidal-like BLA neurons display some form of spike frequency adaptation or accommodation (Washburn and Moises, 1992; Rainnie et al., 1993; Chapman and Chattarji, 2000). Interestingly, this accommodating firing pattern in BLA pyramidal-like neurons is also a salient feature of the firing patterns observed in hippocampal and neocortical pyramidal neurons (Connors and Gutnick, 1990).

Our findings raise a particularly interesting issue concerning the cellular mechanisms by which CIS produced contrasting effects in CA3 and BLA pyramidal neurons that appear to be otherwise similar in terms of their action potential firing patterns and morphological properties. There is evidence for the involvement of excitatory amino acids and NMDA receptors, as well as serotonin, in hippocampal dendritic remodeling (Magarinos and McEwen, 1995b; Magarinos et al., 1999; McEwen, 1999). Serotonin has also been shown to modulate excitatory transmission in the amygdala in a corticosteronedependent manner (Stutzmann et al., 1998). Moreover, glucocorticoids enhance calcium currents in the hippocampus (Kerr et al., 1992; Joels and Vreugdenhil, 1998). Hence, it is quite likely that increased levels of intracellular calcium can act on the dendritic cytoskeleton to trigger structural changes. Thus, the observed differences in dendritic remodeling in BLA neurons may reflect a fundamental difference in the spatiotemporal dynamics of intracellular calcium after stress-induced physiological changes. Indeed, recent electrophysiological studies of amygdaloid long-term potentiation also point to some key differences in mechanisms of synaptic plasticity in the hippocampus and amygdala (Weisskopf and LeDoux, 1999; Weisskopf et al., 1999; Chapman and Chattarji, 2000). These differences in synaptic physiology and plasticity, in turn, can alter the cellular response to the same stressful stimulus and lead to contrasting forms of structural plasticity in the two areas.

\section{Functional implications}

What may be the behavioral consequences of the observed stressinduced morphological changes in the hippocampus and amygdala? Although some studies have reported spatial memory deficits after stress (Luine et al., 1994) or chronic corticosterone treatment (Bodnoff et al., 1995), other studies (Conrad et al., 1999) suggest that stress might also impair memory through non-hippocampal mechanisms, such as enhanced emotionality. Furthermore, stress facilitates classical eye-blink conditioning (Shors et al., 1992), and this facilitation requires activation of NMDA receptors in the BLA (Shors and Mathew, 1998). Corticosterone injections have also been shown to potentiate fear conditioning (Corodimas et al., 1994). A recent interesting study (Conrad et al., 1999), which postulated that chronic stress would 
enhance cued conditioning but not context conditioning, showed that repeated restraint stress facilitates fear conditioning to both context and tone independently of causing hippocampal CA3 dendritic atrophy. The particularly relevant finding of this study was that the atypical antidepressant, tianeptine, failed to prevent enhanced fear conditioning and reduced open-field exploration after stress, although it did prevent neuronal atrophy in the hippocampus. This led the authors to conclude that chronic stress may have a powerful effect on the amygdala, which could override any influence of the hippocampus. Interestingly, CIS, in our study, also caused a significant increase in anxiety-like behavior in the elevated plus-maze. Recent studies have also led to the idea that aversive information relayed from the BLA to a part of the extended amygdala, i.e., the bed nucleus of the stria terminalis, may be involved in anxiety-like behavior (Davis and Shi, 1999). Thus, stress hormones released as a result of stress-induced amygdala activity can strengthen the excitatory drive within the BLA and thereby influence subsequent information processing by the amygdala and its downstream targets. This suggests that chronic stress could lead to an imbalance in HPA axis function through a gradual loss of hippocampal inhibitory control as well as a gain in excitatory control exerted by the amygdala. Therefore, our observations on stress-induced dendritic remodeling in the amygdala may provide a potential cellular substrate for exploring stress-induced disorders that are characterized by diminished cognitive capabilities and abnormally high fear response.

\section{REFERENCES}

Allen JP, Allen CF (1974) Role of the amygdaloid complexes in the stress-induced release of ACTH in the rat. Neuroendocrinology 15:220-230.

Bodnoff SR, Humphreys AG, Lehman JC, Diamond DM, Rose GM, Meaney MJ (1995) Enduring effects of chronic corticosterone treatment on spatial learning, synaptic plasticity, and hippocampal neuropathology in young and mid-aged rats. J Neurosci 15:61-69.

Bremner JD, Randall P, Scott TM, Bronen RA, Seibyl JP, Southwick SM, Delaney RC, McCarthy G, Charney DS, Innis RB (1995) MRI-based measurement of hippocampal volume in patients with combat-related posttraumatic stress disorder. Am J Psychiatry 152:973-981.

Bremner JD, Randall P, Vermetten E, Staib L, Bronen RA, Mazure C, Capelli S, McCarthy G, Innis RB, Charney DS (1997) Magnetic resonance imaging-based measurement of hippocampal volume in posttraumatic stress disorder related to childhood physical and sexual abuse: a preliminary report. Biol Psychiatry 41:23-32.

Chapman PF, Chattarji S (2000) Synaptic plasticity in the amygdala. In: The amygdala, Ed 2 (Aggleton JP, ed), pp 154-177. Oxford: Oxford UP.

Connors BW, Gutnick MJ (1990) Intrinsic firing patterns of diverse neocortical neurons. Trends Neurosci 13:99-104.

Conrad CD, Galea LA, Kuroda Y, McEwen BS (1996) Chronic stress impairs rat spatial memory on the Y maze, and this effect is blocked by tianeptine pretreatment. Behav Neurosci 110:1321-1334.

Conrad CD, LeDoux JE, Magarinos AM, McEwen BS (1999) Repeated restraint stress facilitates fear conditioning independently of causing hippocampal CA3 dendritic atrophy. Behav Neurosci 113:902-913.

Corodimas KP, LeDoux JE, Gold PW, Schulkin J (1994) Corticosterone potentiation of conditioned fear in rats. Ann NY Acad Sci 746:392-393.

Davis M (1992) The role of the amygdala in fear and anxiety. Annu Rev Neurosci 15:353-375.

Davis M, Shi C (1999) The extended amygdala: are the central nucleus of the amygdala and the bed nucleus of the stria terminalis differentially involved in fear versus anxiety? Ann NY Acad Sci 877:281-291.

Davis M, Rainnie D, Cassell M (1994) Neurotransmission in the rat amygdala related to fear and anxiety. Trends Neurosci 17:208-214.

Fitch JM, Juraska JM, Washington LW (1989) The dendritic morphology of pyramidal neurons in the rat hippocampal CA3 area. I. Cell types. Brain Res 479:105-114

Herman JP, Cullinan WE (1997) Neurocircuitry of stress: central control of the hypothalamo-pituitary-adrenocortical axis. Trends Neurosci 20:78-84.

Herman JP, Schafer MK, Young EA, Thompson R, Douglass J, Akil H, Watson SJ (1989) Evidence for hippocampal regulation of neuroen- docrine neurons of the hypothalamo-pituitary-adrenocortical axis. J Neurosci 9:3072-3082.

Jacobson L, Sapolsky R (1991) The role of the hippocampus in feedback regulation of the hypothalamic-pituitary-adrenocortical axis. Endocr Rev 12:118-134.

Joels M, Vreugdenhil E (1998) Corticosteroids in the brain. Cellular and molecular actions. Mol Neurobiol 17:87-108.

Kerr DS, Campbell LW, Thibault O, Landfield PW (1992) Hippocampal glucocorticoid receptor activation enhances voltage-dependent $\mathrm{Ca} 2+$ conductances: relevance to brain aging. Proc Natl Acad Sci USA 89:8527-8531.

LeDoux JE (1994) The amygdala: contributions to fear and stress. Semin Neurosci 6:231-237.

Liang KC, Hon W, Davis M (1994) Pre- and posttraining infusion of $N$-methyl-D-aspartate receptor antagonists into the amygdala impair memory in an inhibitory avoidance task. Behav Neurosci 108:241-253.

Luine V, Villegas M, Martinez C, McEwen BS (1994) Repeated stress causes reversible impairments of spatial memory performance. Brain Res 639:167-170.

Lupien SJ, de Leon M, de Santi S, Convit A, Tarshish C, Nair NP, Thakur M, McEwen BS, Hauger RL, Meaney MJ (1998) Cortisol levels during human aging predict hippocampal atrophy and memory deficits. Nat Neurosci 1:69-73.

Magarinos AM, McEwen BS (1995a) Stress-induced atrophy of apical dendrites of hippocampal CA3c neurons: comparison of stressors. Neuroscience 69:83-88.

Magarinos AM, McEwen BS (1995b) Stress-induced atrophy of apical dendrites of hippocampal CA3c neurons: involvement of glucocorticoid secretion and excitatory amino acid receptors. Neuroscience 69:89-98.

Magarinos AM, McEwen BS, Flugge G, Fuchs E (1996) Chronic psychosocial stress causes apical dendritic atrophy of hippocampal CA3 pyramidal neurons in subordinate tree shrews. J Neurosci 16 : 3534-3540.

Magarinos AM, Deslandes A, McEwen BS (1999) Effects of antidepressants and benzodiazepine treatments on the dendritic structure of CA3 pyramidal neurons after chronic stress. Eur J Pharmacol 371:113-122.

McDonald AJ (1982) Neurons of the lateral and basolateral amygdaloid nuclei: a Golgi study in the rat. J Comp Neurol 212:293-312.

McDonald AJ (1992) Cell types and intrinsic connections of the amygdala. In: The amygdala (Aggleton JP, ed), pp 67-96. New York: Wiley.

McEwen BS (1999) Stress and hippocampal plasticity. Annu Rev Neurosci 22:105-122

Melia KR, Ryabinin AE, Schroeder R, Bloom FE, Wilson MC (1994) Induction and habituation of immediate early gene expression in rat brain by acute and repeated restraint stress. J Neurosci 14:5929-5938.

Nibuya M, Takahashi M, Russell DS, Duman RS (1999) Repeated stress increases catalytic TrkB mRNA in rat hippocampus. Neurosci Lett 267:81-84.

Ortiz J, Fitzgerald LW, Lane S, Terwilliger R, Nestler EJ (1996) Biochemical adaptations in the mesolimbic dopamine system in response to repeated stress. Neuropsychopharmacology 14:443-452.

Pitkanen A, Amaral DG (1994) The distribution of GABAergic cells, fibers, and terminals in the monkey amygdaloid complex: an immunohistochemical and in situ hybridization study. J Neurosci 14:2200-2224

Potvin C, Roff DA (1993) Distribution-free and robust statistical methods: viable alternatives to parametric statistics. Ecology 74:1617-1628.

Rainnie DG, Asprodini EK, Shinnick-Gallagher P (1993) Intracellular recordings from morphologically identified neurons of the basolateral amygdala. J Neurophysiol 69:1350-1362.

Sapolsky RM, Krey LC, McEwen BS (1984) Stress down-regulates corticosterone receptors in a site-specific manner in the brain. Endocrinology 114:287-292.

Sapolsky RM, Zola-Morgan S, Squire LR (1991) Inhibition of glucocorticoid secretion by the hippocampal formation in the primate. J Neurosci 11:3695-3704.

Shankaranarayana Rao BS, Govindaiah, Laxmi TR, Meti BL, Raju TR (2001) Subicular lesions cause dendritic atrophy in CA1 and CA3 pyramidal neurons of the rat hippocampus. Neuroscience 102:319-327.

Sheline YI, Wang PW, Gado MH, Csernansky JG, Vannier MW (1996) Hippocampal atrophy in recurrent major depression. Proc Natl Acad Sci USA 93:3908-3913.

Shors TJ, Mathew PR (1998) NMDA receptor antagonism in the lateral/ basolateral but not central nucleus of the amygdala prevents the induction of facilitated learning in response to stress. Learn Mem 5:220-230.

Shors TJ, Weiss C, Thompson RF (1992) Stress-induced facilitation of classical conditioning. Science 257:537-539.

Sousa N, Lukoyanov NV, Madeira MD, Almeida OF, Paula-Barbosa MM (2000) Reorganization of the morphology of hippocampal neurites and synapses after stress-induced damage correlates with behavioral improvement. Neuroscience 97:253-266.

Starkman MN, Gebarski SS, Berent S, Schteingart DE (1992) Hippocampal formation volume, memory dysfunction, and cortisol levels in patients with Cushing's syndrome. Biol Psychiatry 32:756-765.

Starkman MN, Giordani B, Gebarski SS, Berent S, Schork MA, Schtein- 
gart DE (1999) Decrease in cortisol reverses human hippocampal atrophy following treatment of Cushing's disease. Biol Psychiatry 46: $1595-1602$.

Stutzmann GE, McEwen BS, LeDoux JE (1998) Serotonin modulation of sensory inputs to the lateral amygdala: dependency on corticosterone. J Neurosci 18:9529-9538.

Swanson LW, Petrovich GD (1998) What is the amygdala? Trends Neurosci 21:323-331.

Uno H, Tarara R, Else JG, Suleman MA, Sapolsky RM (1989) Hippocampal damage associated with prolonged and fatal stress in primates. J Neurosci 9:1705-1711.

Vyas A, Mitra R, Chattarji S (2001) Effect of chronic immobilization and unpredictable stress on anxiogenesis. Soc Neurosci Abstr 30:736.13.
Washburn MS, Moises HC (1992) Electrophysiological and morphological properties of rat basolateral amygdaloid neurons in vitro. J Neurosci 12:4066-4079.

Watanabe Y, Gould E, McEwen BS (1992) Stress induces atrophy of apical dendrites of hippocampal CA3 pyramidal neurons. Brain Res 588:341-345.

Weisskopf MG, LeDoux JE (1999) Distinct populations of NMDA receptors at subcortical and cortical inputs to principal cells of the lateral amygdala. J Neurophysiol 81:930-934.

Weisskopf MG, Bauer EP, LeDoux JE (1999) L-type voltage-gated calcium channels mediate NMDA-independent associative long-term potentiation at thalamic input synapses to the amygdala. J Neurosci 19:10512-10519. 